\title{
ОПРЕДЕЛЕНИЕ НАПРЯЖЕНИЙ В ЦИЛИНДРЕ ИЗ КУБИЧЕСКОГО МОНОКРИСТАЛЛА С ПОМОЩЬЮ ИНВЕРСИИ АБЕЛЯ
}

(Представил Х. Абен)

\section{1. Введение}

Определение осесимметричных напряжений:в цилиндре, ось которого совпадает с кристаллографическим направлением [100], методом интегральной фотоупругости рассматривается в $\left[{ }^{1-4}\right]$. Сущность методики, описанной в этих работах, состоит в том, что искомые компоненты тензора напряжений или их разности аппроксимируются степенными или тригонометрическими рядами, содержащими неизвестные коэффициенты. Интегрирование выражений напряжений дает для определения этих неизвестных коэффициентов систему линейных уравнений. Свободными членами полученной линейной системы являются значения разности хода или характеристические величины, т. е. экспериментальные данные. Возможен и другой путь, по которому распределение экспериментальных данных сближается какими-то функциями и из этого распределения с помощью преобразования Абеля находят напряжения. Таким способом определяли напряжения в изотропных цилиндрах $\left[{ }^{5,6}\right]$. Эту же методику широко применяют и для решения других осесимметричных задач экспериментальной механики, например, для определения поля плотностей осесимметричных газовых потоков $\left[{ }^{7}\right]$, исследования различных задач диагностики плазмы $\left[{ }^{8}\right]$ и др.

Настолщая статья посвящена использованию инверсии Абеля для определения осесимметричных напряжений в кубических монокристаллах методом интегральной фотоупругости. Рассматриваются цилиндры, торцы которых параллельны плоскостям (100) или (110).

\section{2. Вывод интегральных уравнений Абеля для цилиндров из кубического монокристалла}

Рассмотрим случай, когда отсутствует градиент напряжений вдоль оси цилиндра (расположение координатных осей показано на рис. 1). В случае цилиндров, торцы которых параллельны плоскостям (110), координатные оси $x, y, z$ совпадают с кристаллографическими направлениями [100], [011] и [110], а для цилиндров, торцы которых параллельны плоскостям (100), оси $x, y, z$ совпадают соответственно с направлениями [001], [010], [100].

Просветим поперечное сечение цилиндра параллельно оси $x_{2}$. Тогда выражение разности хода можно записать в виде $\left[{ }^{9}\right]$

$$
\delta\left(x_{1}\right)=\int_{a}^{b}\left(E_{1} \sigma_{1}+E_{2} \sigma_{2}+E_{3} \sigma_{3}+E_{6} \sigma_{6}\right) \mathrm{d} x_{2},
$$


где $a, b=$ значения координат $x_{2}$ для точек входа и выхода света в поперечное сечение цилиндра. Здесь направление нормальных напряжений $\sigma_{1}, \sigma_{2}, \sigma_{3}$ совпадают с направлениями осей $x_{1}, x_{2}$ и $x_{3}$. Напряжение $\sigma_{6}-$ касательное напряжение $\tau_{1,2}$. Остальные касательные напряжения $\sigma_{4}$ и $\sigma_{5}$ равняются нулю, так как отсутствует градиент напряжений вдоль оси цилиндра. Коэффициенты $E_{i}$ для цилиндров, торцы которых параллельны плоскостям (100), вычисляются по формулам

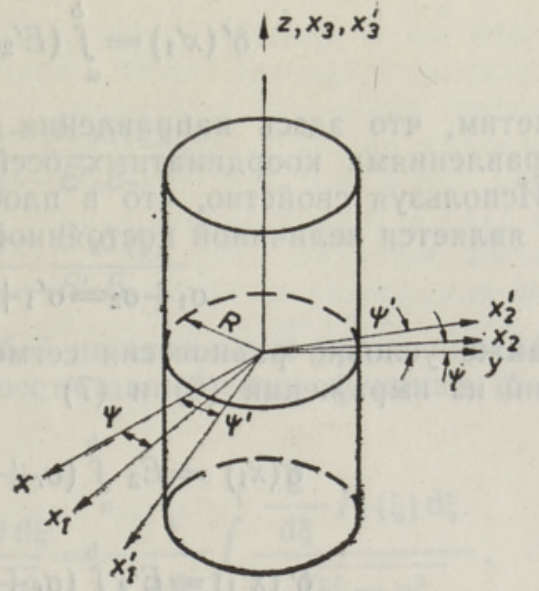

Рис. 1. Расположение координатных осей.

$$
\begin{aligned}
& E_{1}=-C_{11}+\frac{1}{2}\left(C_{11}-C_{44}\right) \sin ^{2} 2 \psi, \\
& E_{2}=-\frac{1}{2}\left(C_{11}-C_{44}\right) \sin ^{2} 2 \psi, \\
& E_{3}=C_{11}
\end{aligned}
$$

и для цилиндров, торцы которых параллельны плоскостям (110), по формулам

$$
\begin{aligned}
& E_{1}=-C_{44}+\frac{1}{2}\left(C_{11}-C_{44}\right) \cos ^{2} \psi\left(1-3 \cos ^{2} \psi\right) \\
& E_{2}=\quad \frac{1}{2}\left(C_{11}-C_{44}\right) \cos ^{2} \psi\left(1-3 \sin ^{2} \psi\right) \\
& E_{3}=C_{44}+\frac{1}{2}\left(C_{11}-C_{44}\right) \cos ^{2} \psi
\end{aligned}
$$

где $C_{11}=\frac{n_{0}^{3}}{2}\left(\pi_{11}-\pi_{12}\right), \quad C_{44}=\frac{n_{0}^{3}}{2} \pi_{44}, \quad n_{0}-$ показатель преломления ненапряженного кристалла.

Учитывая условие равновесия сегмента $\left[{ }^{10}\right]$

$$
\int_{a}^{b} \sigma_{1} \mathrm{~d} x_{2}=0
$$

и то, что касательное напряжение $\sigma_{6}$ является антисимметричной функцией относительно оси $x_{1}$

$$
\int_{a}^{b} \sigma_{6} \mathrm{~d} x_{2}=0
$$

получим из (1)

$$
\delta\left(x_{1}\right)=\int_{a}^{b}\left(E_{2} \sigma_{2}+E_{3} \sigma_{3}\right) \mathrm{d} x_{2} .
$$

Такую же формулу получим при просвечивании цилиндра параллельно оси. $x_{2}^{\prime}$ 


$$
\delta^{\prime}\left(x_{1}^{\prime}\right)=\int_{a}^{b}\left(E_{2}^{\prime} \sigma_{2}^{\prime}+E_{3}^{\prime} \sigma_{3}^{\prime}\right) \mathrm{d} x_{2}^{\prime} .
$$

Отметим, что здесь направления напряжений $\sigma_{2}^{\prime}$ и $\sigma_{3}^{\prime}$ совпадают с направлениями координатных осей $x^{\prime}{ }_{2}$ и $x^{\prime}{ }_{3}$ соответственно.

Используя свойство, что в плоскости сумма нормальных напряжений является величиной постоянной, т. е.

$$
\sigma_{1}+\sigma_{2}=\sigma^{\prime}{ }_{1}+\sigma_{2}^{\prime}=\sigma_{r}+\sigma_{\theta},
$$

а также условие равновесия сегмента (4), получим после преобразований из выражений (6) и (7)

$$
\begin{gathered}
\delta\left(x_{1}\right)=E_{2} \int_{a}^{b}\left(\sigma_{r}+\sigma_{\theta}\right) \mathrm{d} x_{2}+E_{3} \int_{a}^{b} \sigma_{z} \mathrm{~d} x_{2}, \\
\delta^{\prime}\left(x_{1}^{\prime}\right)=E_{2}^{\prime} \int_{a}^{b}\left(\sigma_{r}+\sigma_{\theta}\right) \mathrm{d} x_{2}^{\prime}+E_{-}^{\prime} \int_{a}^{b} \sigma_{z} \mathrm{~d} x_{2}^{\prime} .
\end{gathered}
$$

Иногда предполагают, что в кристаллах $\sigma_{r}, \sigma_{\theta}$ и $\sigma_{z}$ связаны между собой «законом суммы» $\left[{ }^{11,12}\right]$

$$
\sigma_{z}=k\left(\sigma_{n}+\sigma_{\theta}\right),
$$

где $k$ - коэффициент, который в зависимости от условий выращивания и отжига монокристалла может иметь значения $-1,1, \mu, 1 / \mu$ ( $\mu$ - коэффициент Пуассона).

Подставляя соотношение (10) в уравнения (9), получим

$$
\delta^{\prime}\left(x^{\prime}{ }_{1}\right)=\frac{E_{2}^{\prime}+k E_{3}^{\prime}}{E_{2}+k E_{3}} \delta\left(x_{1}\right)
$$

Следовательно, при действии «закона суммы» графики разности хода, полученные при просвечивании параллельно двум произвольным направлениям, должны быть схожими. Например, когда просвечивается поперечное сечение цилиндра из хлористого калия параллельно кристаллографическому направлению [001] $\left(\psi=0^{\circ}\right)$ и под углом $\psi=45^{\circ}$, то

$$
E_{2}=0, \quad E_{3}=2,7 \mathrm{T \Pi a}^{-1}, \quad E_{2}^{\prime}=-5,0 \mathrm{T \Pi a}^{-1}, \quad E_{3}^{\prime}=2,7 \mathrm{T \Pi}^{-1},
$$
и при

$$
\begin{array}{ll}
k=1, & \delta^{\prime}{ }_{45^{\circ}}=-0,48 \delta_{0^{\circ}}, \\
k=-1, & \delta^{\prime}{ }_{45^{\circ}}=2,85 \delta_{0^{\circ}}, \\
k=\mu, & \delta^{\prime}{ }_{45^{\circ}}=-5,17 \delta_{0^{\circ}}, \\
k=\frac{1}{\mu}, & \delta^{\prime}{ }_{45^{\circ}}=0,44 \delta_{0^{\circ}} .
\end{array}
$$

Если провести измерения разности хода на одинаковых расстояниях от оси кристалла $\left(x_{1}=x_{1}^{\prime}\right)$, то выражения (9) можно переписать в виде

$$
\begin{aligned}
& F_{1}(\xi)=2 R \int_{\xi}^{1}, \frac{\left(\sigma_{r}+\sigma_{\theta}\right) \varrho \mathrm{d} \varrho}{\sqrt{\varrho^{2}-\xi^{2}}}, \\
& F_{2}(\xi)=2 R \int_{\xi}^{1} \frac{\sigma_{z} \varrho \mathrm{d} \varrho}{\sqrt{\varrho^{2}-\xi^{2}}},
\end{aligned}
$$


где $\xi=\frac{x_{1}}{R}, \eta=\frac{x_{2}}{R}, \varrho=\frac{r}{R}$,

$$
\begin{aligned}
& F_{1}(\xi)=\frac{E_{3} \delta^{\prime}(\xi)-E^{\prime}{ }_{3} \delta(\xi)}{E_{3} E_{2}^{\prime}-E^{\prime}{ }_{3} E_{2}}, \\
& F_{2}(\xi)=\frac{E_{2} \delta^{\prime}(\xi)-E_{2}^{\prime} \delta(\xi)}{E_{3} E_{2}^{\prime}-E^{\prime}{ }_{3} E_{2}} .
\end{aligned}
$$

Уравнения (14) представляют собой интегральные уравнения Абеля для кубических монокристаллов. Восстанавливаемые функции $\sigma_{r}+\sigma_{\theta}$ и $\sigma_{z}$ выражаются в виде

$$
\begin{gathered}
\left(\sigma_{r}+\sigma_{\theta}\right)=-\frac{1}{\pi R \varrho} \frac{\mathrm{d}}{\mathrm{d} \varrho} \int_{\rho}^{1} \frac{\xi F_{1}(\xi) \mathrm{d} \xi}{\sqrt{\xi^{2}-\varrho^{2}}}=-\frac{1}{\pi R} \int_{\rho}^{1} \frac{\frac{\mathrm{d}}{\mathrm{d} \xi} F_{1}(\xi) \mathrm{d} \xi}{\sqrt{\xi^{2}-\varrho^{2}}}, \\
\sigma_{z}=-\frac{1}{\pi R \varrho} \frac{\mathrm{d}}{\mathrm{d} \varrho} \int_{\rho}^{1} \frac{\xi F_{2}(\xi) \mathrm{d} \xi}{\sqrt{\xi^{2}-\varrho^{2}}}=-\frac{1}{\pi R} \int_{\rho}^{1} \frac{\frac{\mathrm{d}}{\mathrm{d} \xi} F_{2}(\xi) \mathrm{d} \xi}{\sqrt{\xi^{2}-\varrho^{2}}} .
\end{gathered}
$$

Для разделения нормальных напряжений $\sigma_{r}$ и $\sigma_{\theta}$ можно использовать дифференциальное уравнение равновесия

$$
\frac{\partial \sigma_{r}}{\partial \varrho}+\frac{\sigma_{r}-\sigma_{\theta}}{\varrho}=0
$$

откуда

$$
\sigma_{r}(\varrho)=\frac{1}{n^{2}} \int \varrho\left(\sigma_{r}+\sigma_{\theta}\right) \mathrm{d} \varrho+C,
$$

где постоянная интегрирования $C$ определяется из условия

$$
\sigma_{r(\rho=1)}=0 .
$$

\section{3. Примеры аппроксимации экспериментальных данных полиномами}

Экспериментально исследовался цилиндр из монокристалла хлористого калия, торцы которого параллельны плоскостям (100). Длина цилиндра
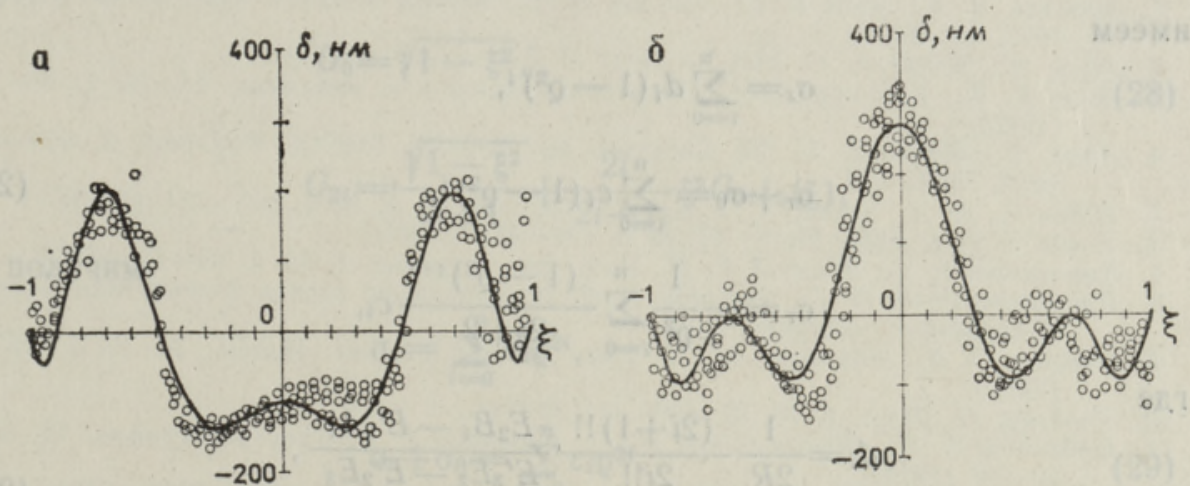

Рис. 2. Распределение разности хода при просвечивании цилиндра параллельно направлениям $\psi=0^{\circ}$ (a) и $\psi=45^{\circ}$ (б). 
$\dot{L}=340$ мм и средний диаметр $\bar{D}=84$ мм. Значения раззностй ходда̇, измеренные в средней части цилиндра, где отсутствовал градиент напряжений вдоль оси цилиндра, приведены на рис. 2. Видно, что распределения разности хода при сканировании поперечного сечения цйлиндра при $\psi=0^{\circ}$ и $\psi=45^{\circ}$ не схожи и, следовательно, согласно формуле (11) в данном случае «закон суммы» не имеет места. Для аппроксимации экспериментальных данных использовались полиномы трех видов и с помощью инверсии Абеля определяли напряжения. Например, если $\delta(\xi)$ и $\delta^{\prime}(\xi)$ аппроксимировать полиномами

$$
\begin{aligned}
& \delta(\xi)=\sum_{i=1}^{n} A_{i}\left(1-\xi^{2}\right)^{i}, \\
& \delta^{\prime}(\xi)=\sum_{i=1}^{n} B_{i}\left(1-\xi^{2}\right)^{i},
\end{aligned}
$$

то из инверсии Абеля получим

$$
\begin{array}{r}
\sigma_{z}=\sum_{i=1}^{n} d_{i} \sqrt{\left(1-\varrho^{2}\right)^{2 i-1}}, \\
\sigma_{r}+\sigma_{\theta}=\sum_{i=1}^{n} c_{i} \sqrt{\left(1-\varrho^{2}\right)^{2 i-1}},
\end{array}
$$

где

$$
\begin{aligned}
& d_{i}=\frac{1}{\pi R} \frac{2 i ! !}{(2 i-1) ! !} \frac{E_{2} B_{i}-E_{2}^{\prime} A_{i}}{E_{3}^{\prime} E_{2}-E_{2}^{\prime} E_{3}}, \\
& c_{i}=\frac{1}{\pi R} \frac{2 i ! !}{(2 i-1) ! !} \frac{E_{3} B_{i}-E_{3}^{\prime} A_{i}}{E_{3} E_{2}^{\prime}-E_{3}^{\prime} E_{2}},
\end{aligned}
$$

и из уравнения равновесия (17)

$$
\sigma_{r}=-\sum_{i=1}^{n} \frac{c_{i}}{2 i+1} \frac{\sqrt{\left(1-\varrho^{2}\right)^{2 i+1}-1}}{\varrho^{2}} .
$$

В случае, когда

$$
\begin{aligned}
& \delta(\xi)=\sqrt{1-\xi^{2}} \sum_{i=0}^{n} A_{i}\left(1-\xi^{2}\right)^{i}, \\
& \delta^{\prime}(\xi)=\sqrt{1-\xi^{2}} \sum_{i=0}^{n} B_{i}\left(1-\xi^{2}\right)^{i}
\end{aligned}
$$

имеем

$$
\begin{aligned}
& \sigma_{z}=\sum_{i=0}^{n} d_{i}\left(1-\varrho^{2}\right)^{i}, \\
& \sigma_{r}+\sigma_{\theta}=\sum_{i=0}^{n} c_{i}\left(1-\varrho^{2}\right)^{i}, \\
& \sigma_{r}=-\frac{1}{\varrho^{2}} \sum_{i=0}^{n} \frac{\left(1-\varrho^{2}\right)^{i+1}}{2 i+2} c_{i},
\end{aligned}
$$

где

$$
\begin{aligned}
& d_{i}=\frac{1}{2 R} \frac{(2 i+1) ! !}{2 i ! !} \frac{E_{2} B_{i}-E_{2}^{\prime} A_{i}}{E_{3}^{\prime} E_{2}-E_{2}^{\prime} E_{3}}, \\
& c_{i}=\frac{1}{2 R} \frac{(2 i+1) ! !}{2 i ! !} \frac{E_{3} B_{i}-E_{3}^{\prime} A_{i}}{E_{3} E_{2}^{\prime}-E_{2} E_{3}^{\prime}}
\end{aligned}
$$



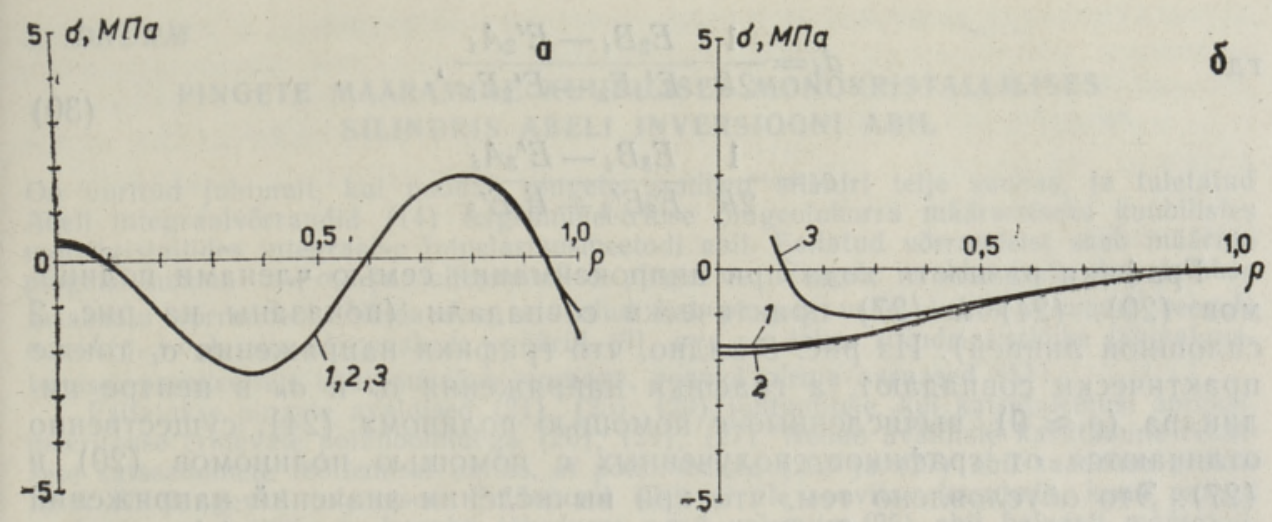

Рис. 3. Распределение напряжений $\sigma_{z}(a)$, $\sigma_{r}(б)$ и $\sigma_{\theta}($ (в) при использовании раз. ных полиномов: $I-(20), 2-(27)$ и $3-(24)$.

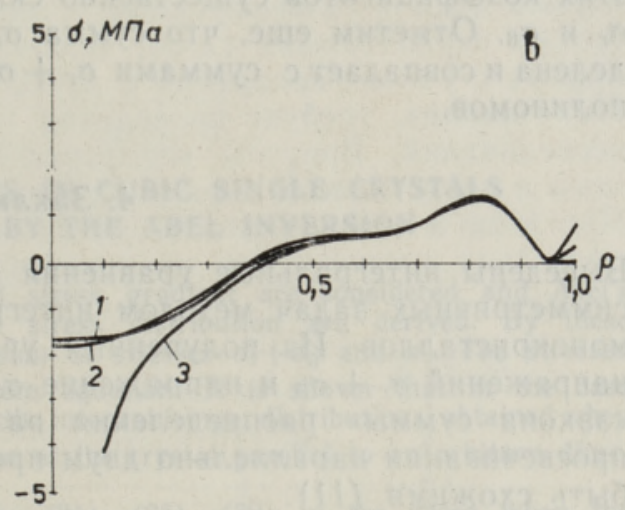

Если применить для приближения разностей хода т. н. $G$-функции $\left[{ }^{13}\right]$

$$
\begin{aligned}
& \delta(\xi)=\sum_{i=0}^{n} A_{i} G_{2 i}, \\
& \delta^{\prime}(\xi)=\sum_{i=0}^{n} B_{i} G_{2 i},
\end{aligned}
$$

где

$$
\begin{aligned}
G_{0} & =\sqrt{1-\xi^{2}} \\
G_{2 i} & =\frac{\sqrt{1-\xi^{2}}}{2 i+1}+\frac{2 i}{2 i+1} \xi^{2} G_{2 i-2}(\xi),
\end{aligned}
$$

то получим

$$
\begin{aligned}
& \sigma_{z}=\sum_{i=0}^{n} d_{i} \mathrm{Q}^{2 i}, \\
& \sigma_{r}+\sigma_{\theta}=\sum_{i=0}^{n} c_{i} \mathrm{Q}^{2 i}, \\
& \sigma_{r}=\sum_{i=0}^{n} \frac{c_{i}}{2 i+2}\left(\mathrm{Q}^{2 i}-1\right),
\end{aligned}
$$

177 


$$
\begin{aligned}
d_{i} & =\frac{1}{2 R} \frac{E_{2} B_{i}-E_{2}^{\prime} A_{i}}{E^{\prime} E_{2}-E_{2}^{\prime} E_{3}}, \\
c_{i} & =\frac{1}{2 R} \frac{E_{3} B_{i}-E^{\prime}{ }_{3} A_{i}}{E_{3} E_{2}^{\prime}-E_{2} E^{\prime}{ }_{3}} .
\end{aligned}
$$

Графики разности хода при аппроксимации семью членами полиномов $(20),(24)$ и (27) практически совпадали (показаны на рис. 2 сплошной линией). Из рис. 3 видно, что графики напряжения $\sigma_{z}$ также практически совпадают, а графики напряжения $\sigma_{r}$ и $\sigma_{\oplus}$ в центре цилиндра $(\varrho \approx 0)$, вычисленные с помощью полинома $(24)$, существенно отличаются от графиков, полученных с помощью. полиномов (20) и (27). Это обусловлено тем, что при вычислении значений напряжений $\sigma_{r}$ и $\sigma_{\theta}$ в точках $\varrho \approx 0$ приходится искать разности коэффициентов, имеющих очень большие значения. Маленькая неточность в величинах этих коэффициентов существенно сказывается в величинах напряжений $\sigma_{r}$ и $\sigma_{\theta}$. Отметим еще, что сумма $\sigma_{r}+\sigma_{\theta}$ в этих точках хорошо определена и совпадает с суммами $\sigma_{r}+\sigma_{\theta}$, полученными с помощью других полиномов.

\section{4. Заключение}

Выведены интегральные уравнения Абеля (14) для исследования осесимметричных залач методом интегральной фотоупругости кубических монокристаллов. Из полученных уравнений можно определить сумму напряжений $\sigma_{r}+\sigma_{\theta}$ и напряжение $\sigma_{z}$. Показано, что в случае действия «закона суммы» распределения разности хода (9). полученные при просвечивании параллельно двум произвольным направлениям, должны быть схожими (11).

Выведены формулы определения напряжений (21), (25) и (29) для случаев, когда экспепиментальные данные апппоксимируются различными полиномами (20), (24) и (27). Использование этих формул при обработке реальных экспепнментальных данных показало, что полиномы (20) и (27) дают практически совпадаюшие результаты, а применение полинома (24) нецелесообразно, так как тогда напряжения $\sigma_{r}$ и $\sigma_{\theta}$ плохо определены в точках $\varrho \approx 0$.

\section{Л ИТЕРАТУРА}

1. Бросман Э. И., Абен Х. К., Каплан М. С. Физика и химия кристаллов. Харьков, 1977, 93-98.

2. Aben, H., Brosman, E. VDI-Berichte, № 313, 45-51 (1978).

3. Абен X. К., Бросман Э. Н. Теоретична приложна механика, 11, № 1, 96-100 (1978).

4. Aben, H., Brosman, E. 7th Congress on Material Testing, Budapest, 2, 409-412, $1978, \quad 409-412$.

5. O'Rourke, R. C.. Saenz, A. W. Quart. Appl. Math., 8, № 3, 303-311 (1950).

6. Doyle, J. F. J. Appl. Mech., 47, № 6, 431-433 (1980).

7. Емельянов В. А., Жаврид Г. П. Инж. физ. журнал, 4, № 4, 64-70 (1962).

8. Преображенский $H . \Gamma$., Пикалов В. В. Неустойчивые задачи диагностики плазмы. Новосибирск. «Наука», 1982. 9. Иднурм С. И., Иозепсон Ю. Н. Изв. АН ЭССР. Физ. Матем., 34, № 2, 23-26
(1985).

10. Poritsku, H. Phys.. 5, № 12, 406-411 (1934).

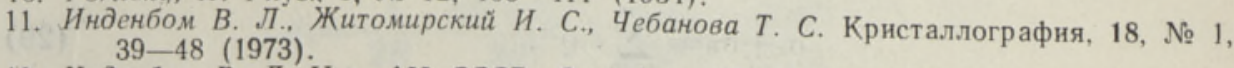

12. Инденбом В. Л. Изв. АН СССР. Сер. физ., 37, № 11, 2258-2267 (1973).

13. Абен Х. К. Интегральная фотоупругость. Таллин, «Валгус», 1975.

Ннститут кибернетики

Академии наук Эстонской ССР
Поступила в редакцию 2/IV 1985 


\section{S. IDNURM}

\section{PINGETE MÄARAMINE KUUBILISES MONOKRISTALLILISES SILINDRIS ABELI INVERSIOONI ABIL}

On uuritud juhtumit, kui puudub pingete gradient silindri telje suunas, ja tuletatud Abeli integraalvõrrandid (14) telgsümmeetrilise pingeolukorra määramiseks kuubilistes monokristallides integraalse fotoclastsusmeetodi abil. Esitatud võrrandeist saab määrata pingete summa $\sigma_{r}+\sigma_{\theta}$ ja teljesuunalise pinge $\sigma_{z}$. Pingete eraldamiseks kasutatakse tasakaalu vōrrandeid. Näidatakse, et juhul kui pingete vahel kehtib «summa reegel» $\sigma_{z}=k\left(\sigma_{r}+\sigma_{\theta}\right)$, siis käiguvahede epüürid (9), mis saadakse silindri ristlooike läbivalgustamisel paralleelselt kahe suvalise suunaga, peavád olema sarnased (11).

Esitatakse pingete avaldised (21), (25), (29) juhtumitele, kui katsetulemusi aproksimeeritakse erinevate polünoomidega $(20),(24),(27)$. Nende avaldiste kasutamine reaalsete katseandmete töötlemisel näitas, et polünoomide $(20)$ ja $(27)$ abil saadakse praktiliselt ühtelangevad tulemused. Polünoomi (24) ei ole soovitav kasutada, kuna pinged $\sigma_{r}$ ja $\sigma_{\theta}$ on ristlōike keskpunkti läheduses $\varrho \approx 0$ valemite $(25)$ abil halvasti määratud.

\section{S. IDNURM}

\section{DETERMINATION OF STRESSES IN CUBIC SINGLE CRYSTALS OF CYLINDRICAL FORM BY THE ABEL INVERSION}

Crystals of cylindrical form without axial stress gradient are considered and Abel's integral equations (14) for axisymmetric stress distribution are derived. By these equations it is possible to determine the sum of stresses $\sigma_{r}+\sigma_{\theta}$ and $\sigma_{z}$. The stresses are separeted by integrating the equilibrium equation. It is shown that in the case when the «sum rule» $\sigma_{z}=k\left(\sigma_{r}+\sigma_{\theta}\right)$ is valid, the integral fringe distributions obtained when the light is passed through the cross-section of the crystal parallel to an arbitary direction, must be similar.

The expressions for stress components $(21),(25),(29)$ in the cases when the experimental data are approximated by polynomes (20), (24), (27), are derived. If we use the polynomes (20) and (27), we get practically the same results. It is not recommended to use the polynome $(24)$, since stresses $\sigma_{r}$ and $\sigma_{\theta}$ by expressions (25) are ill-determined in the central areas $(\varrho \approx 0)$ of the cross-section, 